\title{
Review Article \\ Time- and Event-Driven Communication Process for Networked Control Systems: A Survey
}

\author{
Lei Zou, ${ }^{1}$ Zidong Wang, ${ }^{2,3}$ Hongli Dong, ${ }^{4,5}$ Yurong Liu, ${ }^{3,6}$ and Huijun Gao ${ }^{1,3}$ \\ ${ }^{1}$ Research Institute of Intelligent Control and Systems, Harbin Institute of Technology, Harbin 150001, China \\ ${ }^{2}$ Department of Computer Science, Brunel University, Uxbridge, Middlesex UB8 3PH, UK \\ ${ }^{3}$ King Abdulaziz University, Jeddah 21589, Saudi Arabia \\ ${ }^{4}$ Institute for Automatic Control and Complex Systems, University of Duisburg-Essen, 47057, Germany \\ ${ }^{5}$ College of Electrical and Information Engineering, Northeast Petroleum University, Daqing 163318, China \\ ${ }^{6}$ Department of Mathematics, Yangzhou University, Yangzhou 225002, China
}

Correspondence should be addressed to Zidong Wang; zidong.wang@brunel.ac.uk

Received 9 June 2014; Accepted 8 July 2014; Published 23 July 2014

Academic Editor: Xiao He

Copyright (C) 2014 Lei Zou et al. This is an open access article distributed under the Creative Commons Attribution License, which permits unrestricted use, distribution, and reproduction in any medium, provided the original work is properly cited.

In recent years, theoretical and practical research topics on networked control systems (NCSs) have gained an increasing interest from many researchers in a variety of disciplines owing to the extensive applications of NCSs in practice. In particular, an urgent need has arisen to understand the effects of communication processes on system performances. Sampling and protocol are two fundamental aspects of a communication process which have attracted a great deal of research attention. Most research focus has been on the analysis and control of dynamical behaviors under certain sampling procedures and communication protocols. In this paper, we aim to survey some recent advances on the analysis and synthesis issues of NCSs with different sampling procedures (time- and event-driven sampling) and protocols (static and dynamic protocols). First, these sampling procedures and protocols are introduced in detail according to their engineering backgrounds as well as dynamic natures. Then, the developments of the stabilization, control, and filtering problems are systematically reviewed and discussed in great detail. Finally, we conclude the paper by outlining future research challenges for analysis and synthesis problems of NCSs with different communication processes.

\section{Introduction}

Over the last few decades, developments on networked control systems (NCSs) have led to a growth of attention about the communication of NCSs. In the typical structure of NCSs, all components including sensors, controllers, actuators, and systems to be controlled can transmit information through a shared digital communication network. There are a great deal of advantages of NCSs such as low cost, easy maintenance, and high reliability. Due to the application of digital communication network, the information is firstly sampled into the digital signal before it transmitted over the network, and the communication between different components is implemented subject to the certain protocol.

Sampling is a process of transforming a continuous signal into a discrete one. In practice, sampling is realized by inputting the continuous signal to an analog-to-digital (A/D) converter which can output a sequence of digital signals. Considering the different processes to generate the appropriate sampling intervals (e.g., time-driven sampling and event-driven sampling), we can divide the communication processes of NCSs into two categories: time-driven communication and event-driven communication. In a timedriven communication, the signal is sampled at a sequence of fixed time instants and then transmitted over the network. In an event-driven communication, the signal is sampled only when a certain condition is satisfied. Different communication processes of NCSs can lead to different effects to the closed-loop systems. Hence, it is of both theoretical significance and practical importance to investigate the analysis and synthesis problems for NCSs subject to the time-driven communication and event-driven communication. In this 


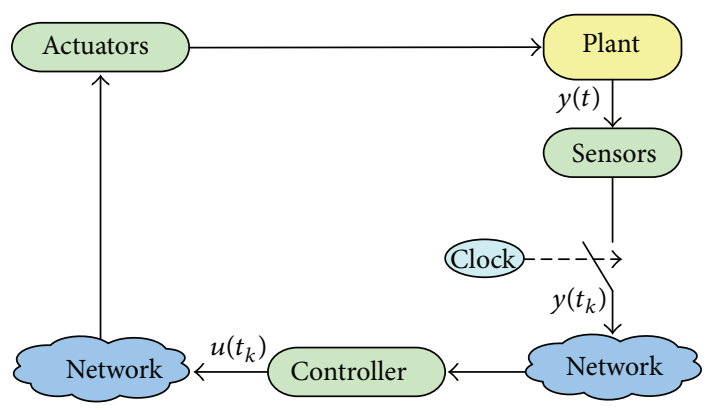

FIGURE 1: General configuration of NCSs with time-driven communication.

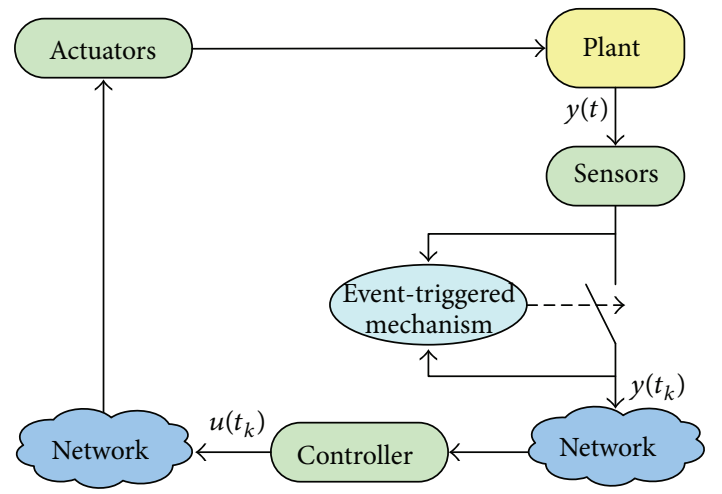

FIGURE 2: General configuration of NCSs with event-triggered sampling.

paper, we focus on the recent advances of NCSs with different sampling procedures and communication protocols.

Time-driven communication is a conventional communication process in which the sampling instants are generated depending on time. Since the time-driven communication is easy to implement in engineering, NCSs with timedriven communication are widely used in practical applications. Generally speaking, time-driven communication is implemented based on three different sampling procedures: periodic sampling procedure, nonuniform sampling procedure, and stochastic sampling procedure. By applying the framework of sampled-data systems, the analysis and synthesis issues have been developed for NCSs with timedriven sampling procedures. Based on the structure of a sampled-data system, a NCS under consideration is divided into a continuous-time plant and a discrete-time controller (or estimator/filter). A general configuration of NCSs with time-driven communication is shown in Figure 1. With the development of digital implementations, sampled-data systems have played an ever-increasingly important role in control engineering practice; see, for example, $[1-4]$ and the references therein.

Event-driven communication is an alternative communication process to time-driven communication aiming to decrease the frequency of sampling and avoid the unnecessary waste of communication and computation resources. In an event-driven communication, the sampling instant is determined as the performance of the system under

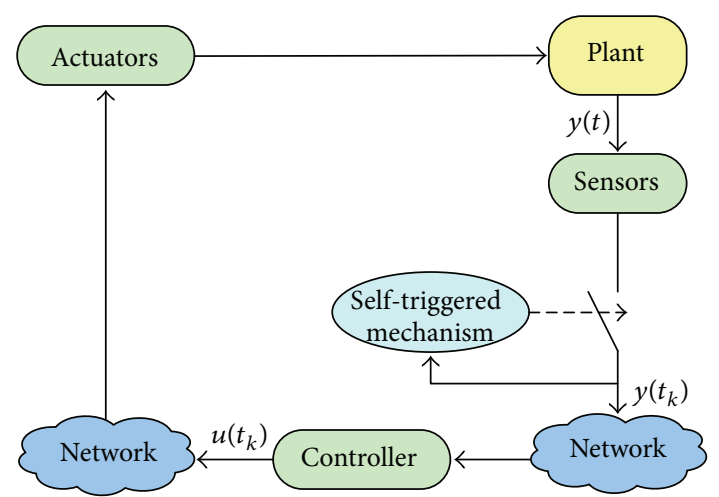

FIGURE 3: General configuration of NCSs with self-triggered sampling.

consideration is getting worse than the requirement (e.g., the system becomes unstable). Recently, the event-driven communication has received increasing attention; see [510]. There are two different sampling schemes in the eventdriven communication: event-triggered sampling and selftriggered sampling. General configurations of NCSs with the event-triggered sampling and the self-triggered sampling are proposed in Figures 2 and 3, respectively. The difference between the event-triggered sampling scheme and the selftriggered sampling scheme is that, in the former scheme, sampling is implemented based on the detection of the "event," while in the later scheme, sampling is implemented according to the prediction for the occurrence of the "event" based on the system model and the current measurement (i.e., the "event" is produced by the prediction).

The focus of this paper is to provide a timely review on the recent theoretical developments of various sampling procedures and communication protocols in time-driven communication and event-driven communication for NCSs. The references discussed in this paper include, but are not limited to the following aspects: (1) NCSs with time-driven sampling procedures, (2) NCSs with event-driven sampling procedures, and (3) NCSs subject to different communication protocols.

The rest of this paper is outlined as follows. In Section 2, the analysis and synthesis problems of NCSs with timedriven sampling procedures are reviewed. Section 3 discusses the analysis and synthesis issues of NCSs with event-driven sampling procedures. Section 4 reviews the developments of NCSs with different communication protocols. The conclusions and future work are given in Section 5.

\section{Time-Driven Sampling Procedures}

In this section, we will recall the recent advances of NCSs with time-driven sampling procedures based on the structure of sampled-data systems.

2.1. Periodic Sampling. Periodic sampling is to sample data with a single rate (i.e., the sampling interval is a constant). As one of the most traditional sampling procedures, a great 
deal of attention has been paid to the analysis and synthesis problems of linear systems, nonlinear systems, neural network, multiagent systems, and stochastic systems subject to periodic sampling.

Stabilization is one of the most fundamental performances of sampled-data systems and has gained particular concerns from many researchers. Based on a periodic sampling procedure, a considerable amount of literature has been published on the stability analysis of sampled-data systems $[1,2,11,12]$. For instance, in [2], the authors have studied the $L_{p}$ input-output stability of the continuous-time closed-loop system under periodic sampling. It has been shown that the closed-loop system could keep $L_{p}$ stable if the original system is $L_{p}$ stable and the sampling period is sufficiently small. Moreover, in [12], the $L_{p}$ stability has been further extended to the globally Lipschitz nonlinear systems with inputs under the periodic sampling procedure. In [11], the stability analysis and controller design have been considered simultaneously for fuzzy systems subject to multirate sampling procedure in which the sampling period was different from the control update period.

Considering the occurrence of incomplete information in NCSs, various attempts have been made to study the control problem under periodic sampling subject to uncertainties [13], packet dropouts [14], and the phenomenon of missing input [15]. For example, in [13], the robust stabilization problem has been investigated for a sampled-data system with nonlinear uncertainties where the periodic sampling setting, data packet dropout, and communication delays are involved together. In [14], the state feedback controller design problem has been dealt with for sampled-data NCSs with networkinduced delays and data packet dropouts by applying a new model of the NCSs. The stabilization problem has been studied for sampled-data control systems with control inputs missing in [15] by using a switched delay system method. Owing to the fact that time delays commonly reside in NCSs and constitute a primary cause of performance degradation or even instability, the control problems and fault detection problems of NCSs with time delays and periodic sampling procedure have been investigated in $[16,17]$, respectively.

With respect to the $H_{\infty}$ control and filtering problems under the periodic sampling procedure, we mention some representative work as follows. In [18], the $H_{\infty}$ and $H_{2}$ control problems have been developed for sampled-data systems concerning the case that the sampling period of sensors was different from the control update period. In [19], the robust $H_{\infty}$ controllers have been developed for a class of uncertain NCSs with periodic sampling, network-induced delays, and packet dropouts based on a linear matrix inequality (LMI) approach. The global output feedback stability method has been proposed in [20] for a class of upper-triangular sampleddata systems with unknown nonlinearities. Backstepping technique is an important approach to designing stabilizing control for a special class of nonlinear systems. In [21], the backstepping technique is employed to stabilize a class of nonlinear systems under periodic sampling.

As a class of complex dynamical networks, neural networks (NNs) have gained particular research interests in the past two decades. In [22], a stable NN-based adaptive control has been studied for a class of multi-input-multioutput (MIMO) nonlinear systems under periodic sampling. Consensus is a significant kind of cooperative behaviors which means that all agents reach an agreement on certain quantities of interest. So far, many efforts have been made to study the consensus problem subject to the periodic sampling procedure [23-26]. For example, the asynchronous consensus problem of second-order agent systems subject to a sampled-data setting and time-varying topology has been investigated in [23]. In [24], the average consensus problem has been studied for multiagent systems where the logarithmic quantization and sampled-data setting are considered simultaneously in communication. On the basis of a delayedinput approach, the average consensus of multiagent studied in [24] can be achieved with a sampling interval which is less than the maximal allowable one.

2.2. Nonuniform Sampling. Periodic sampling is convenient to implement in practical application by setting a constant frequency of $\mathrm{A} / \mathrm{D}$ converter. However, in the NCSs with a limited bandwidth, sampling with the time-varying period could perform better than periodic sampling strategy [27]. For example, in the multisensor tracking task of a highly maneuvering target, the faster sampling is desired during the maneuvering mode than the constant velocity mode. Hence, it is natural that sampling with the time-varying period is more favorable than the system with a periodic sampling in some practical applications. On the other hand, due to the time-varying nature of the channel load, the sampling period might be changed inevitably subject to various reasons. As such, the analysis and synthesis issues for NCSs have been proposed under the framework of nonuniform sampled-data systems in order to cope with the situation that fluctuations in sampling period exist and cannot be ignored.

The problem of stability and stabilization of nonuniform sampled-data systems has received a fast growing research interest in recent years and some results have been reported in the literature; see, for example, [27-30] and the references therein. It is well worth mentioning that there are two main approaches for stability analysis of sampled-data systems with time-varying sampling period. The first one is to model the systems under the nonuniform sampling procedure as a continuous-time system with a delayed control input $[31,32]$. It should be mentioned that the input delay approach has been revised and improved in [32] by applying the scaled small gain theorem. The second approach is to model the closed-loop systems with nonuniform sampling as a hybrid system [33] by taking the impulsive behavior of sampling procedure into consideration.

The issues of $H_{\infty}$ control and filtering for NCSs have also received much research attention [34-37]. For example, in [37], the sampled-data $H_{\infty}$ control and filtering design approach have been addressed for linear systems with a bounded sampling rate. In [35], the $H_{\infty}$ filtering problem has been studied for Itô stochastic systems with the quantized output measurement and nonuniform sampling. By applying a new type of time-dependent Lyapunov function, less conservative results have been proposed in terms of 
LMIs. The fuzzy tracking control problem of nonlinear systems has been investigated in [34] subject to nonuniform sampling and stochastic actuator faults. Genetic regulatory networks (GRNs) play an important role in process of life and attract considerable attention from many researchers in different disciplines. The $H_{\infty}$ filtering problem has been studied in [36] for a class of stochastic GRNs subject to both extrinsic and intrinsic disturbances with the nonuniform sampling setting. Concerning the synchronization problem of complex networks, some results have also been derived with a nonuniform sampling setting; see [38-41].

In $[3,4,42]$, the consensus problem for multiagent systems has been concerned under nonuniform sampling. For instance, the asynchronous consensus problem of continuous-time second-order agent systems has been studied in [3] with fixed topology and time-varying delays subject to the assumption that every sampling interval is bounded.

2.3. Stochastic Sampling. In practical engineering of NCSs, the sampling process is not always implemented in a deterministic way. Due to the effect of networked environment, the sampling interval might change under some random abrupt changes (e.g., random sampler failures). This kind of phenomenon is characterized by stochastic sampling procedure. Stochastic sampling procedure is the further extended phenomenon to the case of nonuniform sampling procedure. In a stochastic sampling setting, there are several constant sampling intervals with known probability of the occurrence of each sampling interval. By applying a transformation of the discrete time instants, the probabilistic sampling system is transformed into a continuous time-delay system with stochastic parameters.

In the past decade, stochastic sampling procedure has been attracting increasing attention both on control problems and on filtering problems [43-47]. For example, the robust $H_{\infty}$ control problem has been concerned in [43] for the linear system with parameter uncertainties and stochastic sampling. A Bernoulli distributed variable has been employed to describe the occurrence of each sampling period. Furthermore, by using a delay system approach, a new stochastic variable has been introduced to characterize the probability of time delay. The same approach has been extended to the $H_{\infty}$ filtering problem [47] and $H_{\infty}$ output tracking control problem [46].

The distributed $H_{\infty}$ filtering problem for sensor networks has been investigated in [48] with a stochastic sampling setting. By applying a transformation, the $H_{\infty}$ filtering problem for stochastic sampled-data system can be converted into a stochastic nonlinear system with multiple bounded timedelays. The same approach has been employed to study the synchronization control problem for dynamical network with stochastic sampling [49]. The estimation problem for neural networks with stochastic sampling has also been studied in [50]. With respect to the Markovian jumping systems, we refer the readers to [51] where the exponential synchronization has been studied for a class of delayed Markovian jumping neural networks with unknown transition probabilities, time-varying delays, and stochastic sampling. The state estimation problem for genetic regulatory networks has also been investigated in [52] with a stochastic sampling setting and time-varying delays.

\section{Event-Driven Sampling Procedures}

3.1. Event-Triggered Sampling. It is a fact that the communication bandwidth and computation resources are limited in practice application. Therefore, it becomes necessary to improve the efficiency of communication channel by avoiding the unnecessary communication. In this case, eventtriggered sampling has been widely applied as an alternative scheme to time-driven sampling. Event-triggered scheme is a special sampling mechanism where the sampling instants are determined in a dynamic way. Different from time-driven sampling, the sampling instants are derived from the "event" which is generated by an event generator. Based on the eventtriggered sampling mechanism, signal is sampled only when a so-called "event-triggered condition" is satisfied. In other words, whether the "event" occurrs depends on the eventtriggered condition. The event-triggered condition can be categorized into two types: fixed threshold condition [53-55] and relative threshold condition $[5,8,10,56]$.

So far, the problems of event-triggered control and filtering have gained a great deal of research attention. There are three representative theoretical frameworks (e.g., the Lyapunov stability theory $[7,8,10]$, hybrid system theory $[5$, $6]$, and input-to-state stability (ISS) theory $[9,57]$ ) to analyze the control and filtering issues with event-triggered sampling scheme. For instance, in [10], a novel event-triggered sampling scheme has been proposed in which the minimum interval between triggered sampling instants is guaranteed. Furthermore, a delay system method has been introduced to deal with the design of controllers of NCSs under such an event-triggered sampling scheme. The same event-triggered sampling scheme and the delay system method have been extended to the $H_{\infty}$ filtering problem of NCSs [7]. The codesign approach of event-triggered sampling scheme and $H_{\infty}$ control has been studied in [8] for NCSs in which the event-triggered condition and controller were designed simultaneously. It can be found that the dynamic of the system under event-triggered sampling scheme would yield the impulsive behavior when the "event" occurred. Therefore, the dynamic process of NCSs under event-triggered sampling scheme can be analyzed based on the structure of the impulsive system; see [5]. In [6], the analysis based on the impulsive system has been improved by transforming the impulsive system into piecewise linear (PWL) model and less conservative results have been derived. In [9], the addressed system has been reformulated as a continuous system with a bounded input which is produced by the eventtriggered sampling scheme. Then, the performance analysis and synthesis issues can be done based on the ISS theory.

Recently, the analysis and synthesis issues of neural network, sensor network, and multiagent systems have been attracting increasing attention due to the advantage that event-triggered sampling scheme can reduce the communication load of the communication channel. In [58], 
the event-triggered state estimation problem for a class of delayed recurrent neural networks has been investigated by using a delayed-input approach. The distributed state estimation problem and $H_{\infty}$ filtering problem have been solved, respectively, in $[59,60]$. With respect to the control problem of multiagent systems, we mention some representative work as follows. The consensus problem for linear multiagent systems has been investigated in [61] via an observer-based controller with the event-triggered sampling scheme. Both centralized event-triggered control and distributed eventtriggered control have been developed based on the outputfeedback control framework. In [62], the distributed control has been designed for multiagent systems to achieve rendezvous based on the event-triggered sampling scheme. In the proposed framework, a new event-triggered sampling scheme has been proposed based on the convergence analysis.

3.2. Self-Triggered Sampling. Event-triggered sampling scheme is implemented with the process of frequent detection of the output signal. This process requires special hardware which may be realized by application-specific integrated circuits (ASIC) or field-programmable gate array (FPGA) processors. As such, a sampling scheme named selftriggered sampling scheme based on a software approach instead of special hardware has been proposed. The concept of self-triggered has been introduced in [63]. In self-triggered sampling scheme, the sampling instant is computed by a function of the data at the previously triggering instant and the dynamic process of the plant.

Self-triggered sampling scheme is developed with the hope to reduce network communication load as well as eventtriggered sampling scheme. The main difference between these two sampling schemes is that, in an event-triggered sampling scheme, the sampling is implemented based on the occurrence of the "event," but in a self-triggered sampling scheme, the sampling is implemented based on the prediction of the occurrence of "event" through the data at the current triggering time and the system model. Some existing results have been reported in [63-68]. For instance, in [64], the behavior of the plant under the virtual event-triggered sampling scheme has been firstly analyzed by applying the ISS theory. Then, the self-triggered sampling scheme for statedependent homogeneous systems has been developed based on the virtual event-triggered sampling scheme and dynamic analysis of the homogeneous systems. In [68], a self-triggered sampling scheme has been proposed to guarantee the finitegain $L_{2}$ stability based on the assumption that the magnitude of the process noise is bounded by a linear function of the norm of the system state. This result has been extended in [67] by relaxing the assumption of the process noise in [68]. The stabilization problem for a class of stochastic systems described by Itô differential equation has been considered in [69] under the self-triggered sampling scheme. By applying Lyapunov approach, the self-triggered sampling scheme has been designed. In [70], the control issue for perturbed nonlinearities subject to bounded external disturbance and small time delay has been dealt with under a self-triggered sampling scheme. For the control problem of the nonlinear systems with norm-bounded parameter uncertainties, disturbances, and sensing/computation/actuation delays, we refer the readers to [71] in which the self-triggered sampling scheme has been designed.

\section{Communication Protocols}

In traditional control systems, the data exchange between different components (e.g., controllers, sensors, and actuators) is implemented by the point-to-point communication. In the point-to-point communication, the components are connected by their relative communication channels. In this case, the communications of all the components in the control systems can be implemented at the same time. Compared with traditional control systems, the communication in NCSs is implemented in a shared network and thereby leading to the communication constraints in communication channels. In a NCS, only one node can make access to the network and be allowed to transmit data per transmission. As such, various communication protocols have been introduced to provide different communication scheduling approaches among nodes.

4.1. Round-Robin and Try-Once-Discard Protocols. The protocols of NCSs can be divided into two categories: static and dynamic protocols. One of the well-known static protocols is round-robin protocol [72]. Round-robin protocol is a class of periodic protocol in which the transmission sequence of nodes is fixed by a predetermined order. In this case, nodes are given access to the network periodically and all the nodes which need to transmit data via network can gain the opportunities to transmit their packets according to a circular sequence. Compared with the static protocol, in dynamic protocols, the opportunities of nodes to transmit data are determined by the states of systems. One of the dynamic protocols is try-once-discard protocol [73]. In the try-oncediscard protocol, the opportunity to transmit data is only assigned to the node with the largest weighted discrepancy between the current and the most recently transmitted value of node's signals [74].

4.2. Analysis and Control under Communication Protocols. For the purpose to analyze the stability of the NCSs subject to certain protocols, the hybrid system theory has been proved to be an effective theory which can be used to deal with the NCSs with either static protocols or dynamic protocols [75-79]. For instance, in [77], a new NCSs model has been developed including varying transmission intervals, varying delays, and communication constraints caused by protocols. Based on the Lyapunov stability theory, the bounds of maximally allowable transmission interval (MATI) and the maximally allowable delay (MAD) have been developed to ensure the stability of the NCSs. The stability of the nonlinear networked control systems has been studied in [80] under a certain communication protocol. The exponential stability can be guaranteed if the MATI satisfies some certain condition. In [76], the stability of NCSs subject to 
communication constraints (caused by certain protocols), time-varying transmission intervals, and time-varying delays has been investigated by using a switched linear uncertain systems approach. It should be mentioned that a framework for the observer design for NCSs with various static and dynamic protocols has been proposed in [78] via an emulation-like approach. Considering the fact that various network induced phenomena would drastically degrade the system performances, a unifying modelling framework has been developed in [81] subject to quantization effects, packet dropouts, time-varying transmission intervals, time-varying transmission delays, and communication protocols. The ISS and $l_{2}$-gain properties of the NCS under consideration have been analyzed based on the proposed modelling framework.

It should be pointed out that most reported results for NCSs with either static or dynamic protocols have been mainly based on the structure of impulsive systems. It might be difficult for this kind of framework to deal with the systems with nonuniform sampling and uncertain transmission delays. As such, a new framework has been studied in [82] to investigate the stability problem of NCSs subject to periodic sampling, transmission delays, and round-robin protocol. The framework proposed in [82] was to reformulate the closed-loop system with round-robin protocol into a switched system with multiple time-varying delays. This approach has been extended in [83] by considering time-vary transmission delays and nonuniform sampling. Furthermore, $L_{2}$-gain analysis has also been studied in [83] based on a timeindependent Lyapunov-Krasovskii functional. The stability analysis for NCSs with packet dropouts has been proposed in [84]. The round-robin protocol and packet dropouts are considered in both two channels (e.g., sensor-to-controller channel and controller-to-actuator channel). Based on the lifting technique, a necessary and sufficient condition of the exponential stability in mean square has been developed. In [85], the authors have proposed an observer-based outputfeedback control subject to round-robin protocol, timevarying transmission intervals, and time-varying delays. It should be mentioned that the observer structure in [85] is a switched one instead of a fixed one.

4.3. The Codesign Issue under Communication Protocols. On another research front, the codesign problem of protocol and controller has also gained particular attentions. In [74], the try-once-discard protocol and the discrete-time controller have been designed simultaneously in terms of matrix inequalities. In [86], the $H_{\infty}$ controller has been designed as well as the try-once-discard protocol for a class of NCSs with random time delay. Based on the Lyapunov function method and switched system theory, a sufficient condition has been derived in [86] to guarantee the asymptotic stability of the closed-loop system with the try-once-discard protocol proposed. The codesign problem of protocol and observer has been studied in [87] for a class of linear timeinvariant systems. Sufficient conditions have been derived for the existence of the proposed protocol and observer via matrix inequalities. In [79], the codesign issue for a class of NCSs with communication protocols and periodic sampling scheme has been studied by applying a new compensation scheme and discrete-time switched systems approach.

\section{Conclusion and Future Work}

In this paper, we have reviewed some developments of the analysis and synthesis problems of NCSs subject to different communication processes. According to different sampling procedures, we have divided the communication processes into two categories: time-driven communication and eventdriven communication. Based on this, various stability analysis, control, filtering, and protocol design issues have been surveyed in great detail. Based on the literature review, some related topics for the future research work are listed as follows.

(1) The problems of distributed control and filtering with a certain sampling approach and communication protocol are of engineering significance. It would be an interesting research topic to analyze the dynamical behaviors of sensor networks, neural networks, and multiagent systems subject to different kinds of communication processes.

(2) It would be meaningful to study the problems of control and filtering for time-varying systems subject to a certain communication protocol over a finite time horizon.

(3) For the analysis and synthesis issues subject to a given communication protocol, the systems under consideration are continuous systems in most of the existing results. Consequently, it makes sense to investigate the analysis and synthesis issues for nonlinear discrete-time systems subject to a given communication protocol.

(4) Since the transmission interval (fixed by the communication protocol) is always much smaller than the sampling interval in practice applications, it is necessary to consider the dynamical behaviors with a "slow" sampling scheme and a "fast" communication protocol.

(5) A practical engineering application of the existing theories and methodologies would be the batch reactor control system.

(6) The existing results on time- and event-driven communication process for networked control systems can be extended to more general systems such as complex systems and multiagent systems with networkinduced problems [88-97] by using more up-to-date algorithms [98-102].

\section{Conflict of Interests}

The authors declare that there is no conflict of interests regarding the publication of this paper.

\section{Acknowledgments}

This work was supported in part by the National Natural Science Foundation of China under Grants 61329301, 61374127, 
and 61374010, the Royal Society of the UK, and the Alexander von Humboldt Foundation of Germany.

\section{References}

[1] D. S. Bernstein and C. V. Hollot, "Robust stability for sampleddata control systems," Systems \& Control Letters, vol. 13, no. 3, pp. 217-226, 1989.

[2] T. Chen and B. A. Francis, "Input-output stability of sampleddata systems," IEEE Transactions on Automatic Control, vol. 36, no. 1, pp. 50-58, 1991.

[3] Y. Gao and L. Wang, "Asynchronous consensus of continuoustime multi-agent systems with intermittent measurements," International Journal of Control, vol. 83, no. 3, pp. 552-562, 2010.

[4] J. Wu, Y. Shi, and H. Li, "Consensus in multi-agent systems with non-uniform sampling," in Proceedings of the American Control Conference (ACC '13), pp. 3260-3265, Washington, Wash, USA, June 2013.

[5] M. C. F. Donkers and W. P. M. H. Heemels, "Output-based event-triggered control with guaranteed $L_{\infty}$-gain and improved and decentralized event-triggering," IEEE Transactions on Automatic Control, vol. 57, no. 6, pp. 1362-1376, 2012.

[6] W. P. M. Heemels, M. C. F. Donkers, and A. R. Teel, "Periodic event-triggered control for linear systems," IEEE Transactions on Automatic Control, vol. 58, no. 4, pp. 847-861, 2013.

[7] S. Hu and D. Yue, "Event-based $H_{\infty}$ filtering for networked system with communication delay," Signal Processing, vol. 92, no. 9, pp. 2029-2039, 2012.

[8] C. Peng and T. C. Yang, "Event-triggered communication and $H_{\infty}$ control co-design for networked control systems," Automatica, vol. 49, no. 5, pp. 1326-1332, 2013.

[9] P. Tabuada, "Event-triggered real-time scheduling of stabilizing control tasks," IEEE Transactions on Automatic Control, vol. 52, no. 9, pp. 1680-1685, 2007.

[10] D. Yue, E. Tian, and Q. Han, "A delay system method for designing event-triggered controllers of networked control systems," IEEE Transactions on Automatic Control, vol. 58, no. 2, pp. 475-481, 2013.

[11] D. W. Kim, J. B. Park, Y. H. Joo, and S. H. Kim, "Multirate digital control for fuzzy systems: LMI-based design and stability analysis," International Journal of Control, Automation and Systems, vol. 4, no. 4, pp. 506-515, 2006.

[12] L. Zaccarian, A. R. Teel, and D. Nešić, "On finite gain $L_{p}$ stability of nonlinear sampled-data systems," Systems and Control Letters, vol. 49, no. 3, pp. 201-212, 2003.

[13] M. Yu, L. Wang, and T. Chu, "Sampled-data stabilisation of networked control systems with nonlinearity," IEE Proceedings: Control Theory and Applications, vol. 152, no. 6, pp. 609-614, 2005.

[14] D. Yue, Q.-L. Han, and C. Peng, "State feedback controller design of networked control systems," IEEE Transactions on Circuits and Systems II: Express Briefs, vol. 51, no. 11, pp. 640644, 2004.

[15] W. A. Zhang and L. Yu, "Stabilization of sampled-data control systems with control inputs missing," IEEE Transactions on Automatic Control, vol. 55, no. 2, pp. 447-452, 2010.

[16] L. Hetel, J. Daafouz, J. P. Richard, and M. Jungers, "Delaydependent sampled data control based on delay estimates," Systems and Control Letters, vol. 60, no. 2, pp. 146-150, 2011.

[17] P. Zhang, S. X. Ding, G. Z. Wang, and D. H. Zhou, "Fault detection for multirate sampled-data systems with time delays,"
International Journal of Control, vol. 75, no. 18, pp. 1457-1471, 2002.

[18] T. Chen and L. Qiu, " $H_{\infty}$ design of general multirate sampleddata control systems," Automatica, vol. 30, no. 7, pp. 1139-1152, 1994.

[19] D. Yue, Q.-L. Han, and J. Lam, "Network-based robust $H_{\infty}$ control of systems with uncertainty," Automatica, vol. 41, no. 6, pp. 999-1007, 2005.

[20] H. Du, C. Qian, Y. He, and Y. Cheng, "Global sampled-data output feedback stabilisation of a class of upper-triangular systems with input delay," IET Control Theory \& Applications, vol. 7, no. 10, pp. 1437-1446, 2013.

[21] B. Wu and Z. Ding, "Asymptotic stabilisation of a class of nonlinear systems via sampled-data output feedback control," International Journal of Control, vol. 82, no. 9, pp. 1738-1746, 2009.

[22] F. Sun, Z. Sun, and P.-Y. Woo, "Stable neural-network-based adaptive control for sampled-data nonlinear systems," IEEE Transactions on Neural Networks, vol. 9, no. 5, pp. 956-968, 1998.

[23] Y. Gao and L. Wang, "Sampled-data based consensus of continuous-time multi-agent systems with time-varying topology," IEEE Transactions on Automatic Control, vol. 56, no. 5, pp. 1226-1231, 2011.

[24] S. Liu, T. Li, L. Xie, M. Fu, and J. Zhang, "Continuous-time and sampled-data-based average consensus with logarithmic quantizers," Automatica, vol. 49, no. 11, pp. 3329-3336, 2013.

[25] Z. J. Tian, T. Z. Huang, J. L. Shao, and J.-P. Hu, "Leader-following consensus for multi-agent systems via sampled-data control," IET Control Theory and Applications, vol. 5, no. 14, pp. 16581665, 2011.

[26] D. Xie and J. Chen, "Consensus problem of data-sampled networked multi-agent systems with time-varying communication delays," Transactions of the Institute of Measurement and Control, vol. 35, no. 6, pp. 753-763, 2013.

[27] Y. S. Suh, "Stability and stabilization of nonuniform sampling systems," Automatica, vol. 44, no. 12, pp. 3222-3226, 2008.

[28] H. Gao and T. Chen, "Stabilization of nonlinear systems under variable sampling: a fuzzy control approach," IEEE Transactions on Fuzzy Systems, vol. 15, no. 5, pp. 972-983, 2007.

[29] J. M. Olm, G. A. Ramos, and R. Costa-Castelló, "Stability analysis of digital repetitive control systems under time-varying sampling period," IET Control Theory \& Applications, vol. 5, no. 1, pp. 29-37, 2011.

[30] J. Tsinias, "Sampled-data feedback practical semi-global controllability and stabilization for time-varying systems," International Journal of Control, vol. 80, no. 1, pp. 21-34, 2007.

[31] E. Fridman, A. Seuret, and J. Richard, "Robust sampleddata stabilization of linear systems: an input delay approach," Automatica, vol. 40, no. 8, pp. 1441-1446, 2004.

[32] L. Mirkin, "Some remarks on the use of time-varying delay to model sample-and-hold circuits," IEEE Transactions on Automatic Control, vol. 52, no. 6, pp. 1109-1112, 2007.

[33] P. Naghshtabrizi, J. P. Hespanha, and A. R. Teel, "On the robust stability and stabilization of sampled-data systems: a hybrid system approach," in Proceedings of the 45th IEEE Conference on Decision and Control (CDC '06), pp. 4873-4878, San Diego, Calif, USA, December 2006.

[34] S. Hu, D. Yue, Z. Du, and J. Liu, "Reliable $H_{\infty}$ non-uniform sampling tracking control for continuous-time non-linear systems with stochastic actuator faults," IET Control Theory of Applications, vol. 6, no. 1, pp. 120-129, 2012. 
[35] M. Liu, J. You, and X. Ma, " $H_{\infty}$ filtering for sampled-data stochastic systems with limited capacity channel," Signal Processing, vol. 91, no. 8, pp. 1826-1837, 2011.

[36] B. Shen, Z. Wang, J. Liang, and X. Liu, "Sampled-data $H_{\infty}$ filtering for stochastic genetic regulatory networks," International Journal of Robust and Nonlinear Control, vol. 21, no. 15, pp. 17591777, 2011.

[37] V. Suplin, E. Fridman, and U. Shaked, "Sampled-data $H_{\infty}$ control and filtering: nonuniform uncertain sampling," Automatica, vol. 43, no. 6, pp. 1072-1083, 2007.

[38] Q. Gan and Y. Liang, "Synchronization of chaotic neural networks with time delay in the leakage term and parametric uncertainties based on sampled-data control," Journal of the Franklin Institute, vol. 349, no. 6, pp. 1955-1971, 2012.

[39] T. H. Lee, Z.-G. Wu, and J. H. Park, "Synchronization of a complex dynamical network with coupling time-varying delays via sampled-data control," Applied Mathematics and Computation, vol. 219, no. 3, pp. 1354-1366, 2012.

[40] N. Li, Y. Zhang, J. Hu, and Z. Nie, "Synchronization for general complex dynamical networks with sampled-data," Neurocomputing, vol. 74, no. 5, pp. 805-811, 2011.

[41] S. J. S. Theesar, S. Banerjee, and P. Balasubramaniam, "Synchronization of chaotic systems under sampled-data control," Nonlinear Dynamics, vol. 70, no. 3, pp. 1977-1987, 2012.

[42] G. Wen, Z. Duan, W. Yu, and G. Chen, "Consensus of multiagent systems with nonlinear dynamics and sampled-data information: a delayed-input approach," International Journal of Robust and Nonlinear Control, vol. 23, no. 6, pp. 602-619, 2013.

[43] H. Gao, J. Wu, and P. Shi, "Robust sampled-data H8 control with stochastic sampling," Automatica, vol. 45, no. 7, pp. 1729-1736, 2009.

[44] T. H. Lee, J. H. Park, S. M. Lee, and O. M. Kwon, "Robust synchronisation of chaotic systems with randomly occurring uncertainties via stochastic sampled-data control," International Journal of Control, vol. 86, no. 1, pp. 107-119, 2013.

[45] Y. Li, Q. Zhang, J. Ren, and H. Lv, "Stabilization for networked control systems with random sampling periods and random delays by Markov chains," in Proceedings of the 32nd Chinese Control Conference (CCC '13), pp. 6594-6599, Xi'an, China, July 2013.

[46] S. Wen and Z. Zeng, "Robust sampled-data $H_{\infty}$ output tracking control for a class of nonlinear networked systems with stochastic sampling," International Journal of Systems Science, vol. 44, no. 9, pp. 1626-1638, 2013.

[47] J. Wu, X. Chen, and H. Gao, "Ho filtering with stochastic sampling," Signal Processing, vol. 90, no. 4, pp. 1131-1145, 2010.

[48] B. Shen, Z. Wang, and X. Liu, "A stochastic sampled-data approach to distributed $H_{\infty}$ filtering in sensor networks," IEEE Transactions on Circuits and Systems I: Regular Papers, vol. 58, no. 9, pp. 2237-2246, 2011.

[49] B. Shen, Z. Wang, and X. Liu, "Sampled-data synchronization control of dynamical networks with stochastic sampling," IEEE Transactions on Automatic Control, vol. 57, no. 10, pp. 26442650, 2012.

[50] T. H. Lee, J. H. Park, O. M. Kwon, and S. M. Lee, "Stochastic sampled-data control for state estimation of time-varying delayed neural networks," Neural Networks, vol. 46, pp. 99-108, 2013.

[51] A. Chandrasekar, R. Rakkiyappan, F. A. Rihan, and S. Lakshmanan, "Exponential synchronization of Markovian jumping neural networks with partly unknown transition probabilities via stochastic sampled-data control," Neurocomputing, vol. 133, no. 10, pp. 385-398, 2014.

[52] T. H. Lee, M. J. Park, O. M. Kwon, J. H. Park, and S. M. Lee, "State estimation for genetic regulatory networks with time-varying delay using stochastic sampled-data," in Proceedings of the 9th Asian Control Conference, pp. 1-6, Istanbul, Turkey, 2013.

[53] E. Garcia and P. J. Antsaklis, "Model-based event-triggered control with time-varying network delays," in Proceedings of the 50th IEEE Conference on Decision and Control and European Control Conference (CDC-ECC '11), pp. 1650-1655, Orlando, Fla, USA, December 2011.

[54] M. Miskowicz, "Send-on-delta concept: an event-based data reporting strategy," Sensors, vol. 6, no. 1, pp. 49-63, 2006.

[55] V. H. Nguyen and Y. S. Suh, "Networked estimation for eventbased sampling systems with packet dropouts," Sensors, vol. 9, no. 4, pp. 3078-3089, 2009.

[56] H. Yu and P. J. Antsaklis, "Event-triggered output feedback control for networked control systems using passivity: achieving $L_{2}$ stability in the presence of communication delays and signal quantization," Automatica, vol. 49, no. 1, pp. 30-38, 2013.

[57] C. de Persis, R. Sailer, and F. Wirth, "Parsimonious eventtriggered distributed control: a Zeno free approach," Automatica, vol. 49, no. 7, pp. 2116-2124, 2013.

[58] H. Li, "Event-triggered state estimation for a class of delayed recurrent neural networks with sampled-data information," Abstract and Applied Analysis, vol. 2012, Article ID 731453, 21 pages, 2012.

[59] X. Ge, Q.-L. Han, F. Yang, and X.-M. Zhang, "Distributed eventtriggered $H_{\infty}$ filtering over sensor networks with coupling delays," in Proceedings of the 39th Annual Conference of the IEEE Industrial-Electronics-Society, pp. 5074-5079, Vienna, Austria, 2013.

[60] L. Yan, X. Zhang, Z. Zhang, and Y. Yang, "Distributed state estimation in sensor networks with event-triggered communication," Nonlinear Dynamics, vol. 76, no. 1, pp. 169-181, 2014.

[61] H. Zhang, G. Feng, H. Yan, and Q. Chen, "Observer-based output feedback event-triggered control for consensus of multiagent systems," IEEE Transactions on Industrial Electronics, vol. 61, no. 9, pp. 4885-4894, 2014.

[62] Y. Fan, G. Feng, Y. Wang, and C. Song, "Distributed eventtriggered control of multi-agent systems with combinational measurements," Automatica, vol. 49, no. 2, pp. 671-675, 2013.

[63] M. Velasco, J. Fuertes, and P. Marti, "The self triggered task model for real-time control systems," in Proceedings of the Work in Progress Proceedings of the 24th IEEE Real-Time Systems Symposium, pp. 67-70, 2003.

[64] A. Anta and P. Tabuada, "To sample or not to sample: selftriggered control for nonlinear systems," IEEE Transactions on Automatic Control, vol. 55, no. 9, pp. 2030-2042, 2010.

[65] A. Anta and P. Tabuada, "Isochronous manifolds in selftriggered control," in Proceedings of the 48th IEEE Conference on Decision and Control held jointly with 2009 28th Chinese Control Conference (CDC/CCC '09), pp. 3194-3199, Shanghai, China, December 2009.

[66] M. Lemmon, T. Chantem, X. S. Hu, and Z. Matthew, "On selftriggered full-information $H_{\infty}$ controllers," in Proceedings of the 10th International Conference on Hybrid Systems: Computation and Control, pp. 371-384, Pisa, Italy, 2007.

[67] X. Wang and M. D. Lemmon, "Self-triggering under stateindependent disturbances," IEEE Transactions on Automatic Control, vol. 55, no. 6, pp. 1494-1500, 2010. 
[68] X. Wang and M. D. Lemmon, "Self-triggered feedback control systems with finite-gain $L_{2}$ stability," IEEE Transactions on Automatic Control, vol. 54, no. 3, pp. 452-467, 2009.

[69] W. Aggoune, B. Castillo, and S. Di Gennaro, "Self-triggered robust control of nonlinear stochastic systems," in Proceedings of the 51st IEEE Conference on Decision and Control (CDC '12), pp. 7547-7552, Maui, Hawaii, USA, December 2012.

[70] U. Tiberi and K. H. Johansson, "A simple self-triggered sampler for perturbed nonlinear systems," Nonlinear Analysis: Hybrid Systems, vol. 10, pp. 126-140, 2013.

[71] M. D. Di Benedetto, S. Di Gennaro, and A. D’Innocenzo, "Digital self-triggered robust control of nonlinear systems," International Journal of Control, vol. 86, no. 9, pp. 1664-1672, 2013.

[72] S. Lall and G. Dullerud, "An LMI solution to the robust synthesis problem for multi-rate sampled-data systems," Automatica, vol. 37, no. 12, pp. 1909-1922, 2001.

[73] G. C. Walsh, H. Ye, and L. G. Bushnell, "Stability analysis of networked control systems," IEEE Transactions on Control Systems Technology, vol. 10, no. 3, pp. 438-446, 2002.

[74] D. B. Dačić and D. Nešić, "Quadratic stabilization of linear networked control systems via simultaneous protocol and controller design," Automatica, vol. 43, no. 7, pp. 1145-1155, 2007.

[75] D. Antunes, J. P. Hespanha, and C. Silvestre, "Stochastic networked control systems with dynamic protocols," in Proceedings of the American Control Conference (ACC '11), pp. 1686-1691, San Francisco, Calif, USA, July 2011.

[76] M. C. F. Donkers, W. P. M. H. Heemels, N. van de Wouw, and L. Hetel, "Stability analysis of networked control systems using a switched linear systems approach," IEEE Transactions on Automatic Control, vol. 56, no. 9, pp. 2101-2115, 2011.

[77] W. P. M. H. Heemels, A. R. Teel, N. van de Wouw, and D. Nešić, "Networked control systems with communication constraints: tradeoffs between transmission intervals, delays and performance," IEEE Transactions on Automatic Control, vol. 55, no. 8, pp. 1781-1796, 2010.

[78] R. Postoyan and D. Nesic, "A framework for the observer design for networked control systems," IEEE Transactions on Automatic Control, vol. 57, no. 5, pp. 1309-1314, 2012.

[79] H. Song, L. Yu, and W.-A. Zhang, "Stabilisation of networked control systems with communication constraints and multiple distributed transmission delays," IET Control Theory \& Applications, vol. 3, no. 10, pp. 1307-1316, 2009.

[80] G. C. Walsh, O. Beldiman, and L. G. Bushnell, "Asymptotic behavior of nonlinear networked control systems," Institute of Electrical and Electronics Engineers. Transactions on Automatic Control, vol. 46, no. 7, pp. 1093-1097, 2001.

[81] S. J. L. van Loon, M. C. F. Donkers, N. van de Wouw, and W. P. M. Heemels, "Stability analysis of networked and quantized linear control systems," Nonlinear Analysis: Hybrid Systems, vol. 10, pp. 111-125, 2013.

[82] K. Liu, E. Fridman, L. Hetel, and J.-P. Richard, "Sampled-data stabilization via round-robin scheduling: a direct LyapunovKrasovskii approach," in Proceedings of the 18th World Congress of the International Federation of Automatic Control, pp. 14591464, Milano, Italy, September 2011.

[83] K. Liu, E. Fridman, and L. Hetel, "Stability and $L_{2}$-gain analysis of networked control systems under round-robin scheduling: a time-delay approach," Systems and Control Letters, vol. 61, no. 5, pp. 666-675, 2012.
[84] Y. Xu, H. Su, Y. J. Pan, Z. Wu, and W. Xu, "Stability analysis of networked control systems with round-robin scheduling and packet dropouts," Journal of the Franklin Institute, vol. 350, no. 8, pp. 2013-2027, 2013.

[85] N. W. Bauer, M. C. F. Donkers, N. van de Wouw, and W. P. M. H. Heemels, "Decentralized observer-based control via networked communication," Automatica, vol. 49, no. 7, pp. 2074-2086, 2013.

[86] C. Zhou, M. Du, and Q. Chen, "Co-design of dynamic scheduling and $H_{\infty}$ control for networked control systems," Applied Mathematics and Computation, vol. 218, no. 21, pp. 10767-10775, 2012.

[87] D. Dačić and D. Nešić, "Observer design for wired linear networked control systems using matrix inequalities," Automatica, vol. 44, no. 11, pp. 2840-2848, 2008.

[88] M. Darouach and H. S. Ali, "Optimal unbiased functional filtering in the frequency domain," Systems Science and Control Engineering, vol. 2, no. 1, pp. 308-315, 2014.

[89] Z. Ding, G. Wei, and X. Ding, "Speed identification and control for permanent magnet synchronous motor via sliding mode approach," Systems Science and Control Engineering, vol. 2, no. 1, pp. 161-167, 2014.

[90] J. Liang, F. Sun, and X. Liu, "Finite-horizon $H_{\infty}$ filtering for time-varying delay systems with randomly varying nonlinearities and sensor saturations," Systems Science and Control Engineering, vol. 2, no. 1, pp. 108-118, 2014.

[91] Q. Liu, Z. Wang, X. He, and D. H. Zhou, "A survey of eventbased strategies on control and estimation," Systems Science and Control Engineering, vol. 2, no. 1, pp. 90-97, 2014.

[92] B. Munyazikwiye, K. Robbersmyr, and H. Karimi, "A statespace approach to mathematical modeling and parameters identification of vehicle frontal crash," Systems Science and Control Engineering, vol. 2, no. 1, pp. 351-361, 2014.

[93] L. Qin, X. He, and D. H. Zhou, "A survey of fault diagnosis for swarm systems," Systems Science and Control Engineering, vol. 2, no. 1, pp. 13-23, 2014.

[94] B. Shen, "A survey on the applications of the Krein-space theory in signal estimation," Systems Science and Control Engineering, vol. 2, no. 1, pp. 143-149, 2014.

[95] J. Shen and J. Lam, "Decay rate constrained stability analysis for positive systems with discrete and distributed delays," Systems Science and Control Engineering, vol. 2, no. 1, pp. 7-12, 2014.

[96] Q. Wang, H. Gao, F. Alsaadi, and T. Hayat, "An overview of consensus problems in constrained multi-agent coordination," Systems Science and Control Engineering, vol. 2, no. 1, pp. 275284, 2014.

[97] X. Wang, E. Yaz, and J. Long, "Robust and resilient statedependent control of continuous-time nonlinear systems with general performance criteria," Systems Science and Control Engineering, vol. 2, no. 1, pp. 34-40, 2014.

[98] N. Li, H.-Y. Sun, and Q.-L. Zhang, "The dynamics and bifurcation control of a singular biological economic model," International Journal of Automation and Computing, vol. 9, no. 1, pp. 1-7, 2012.

[99] Q. Liang, Y.-Z. Wang, and Y.-H. Zhang, "Resource virtualization model using hybrid-graph representation and converging algorithm for cloud computing," International Journal of Automation and Computing, vol. 10, no. 6, pp. 597-606, 2013.

[100] H.-P. Ma, X.-Y. Ruan, and Z.-X. Pan, "Handling multiple objectives with biogeography-based optimization," International Journal of Automation and Computing, vol. 9, no. 1, pp. 30-36, 2012. 
[101] M. S. Kumar and P. Philominathan, "Purcell's swimmer revisited," International Journal of Automation and Computing, vol. 9, no. 3, pp. 325-330, 2012.

[102] X.-Y. Zhang and C.-L. Zhou, "From biological consciousness to machine consciousness: an approach to make smarter machines," International Journal of Automation and Computing, vol. 10, no. 6, pp. 498-505, 2013. 


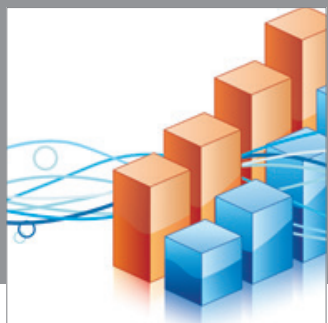

Advances in

Operations Research

mansans

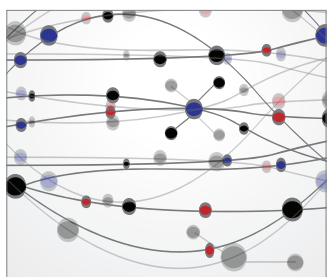

The Scientific World Journal
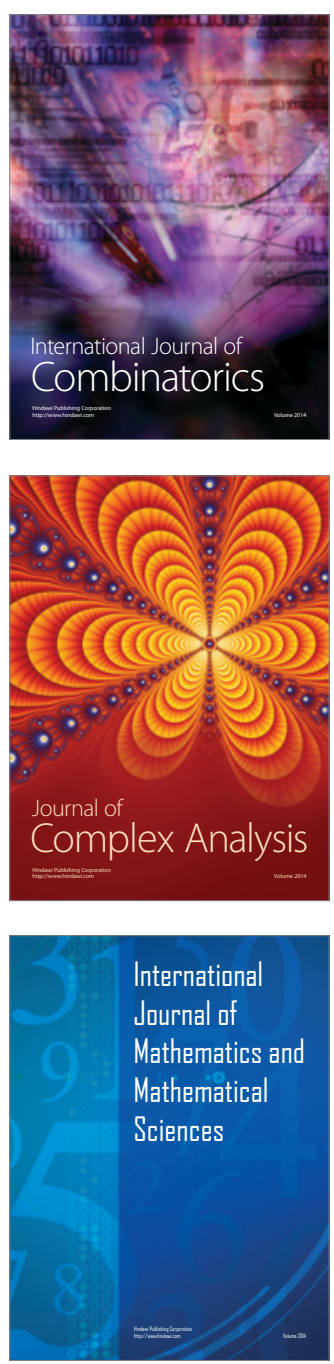
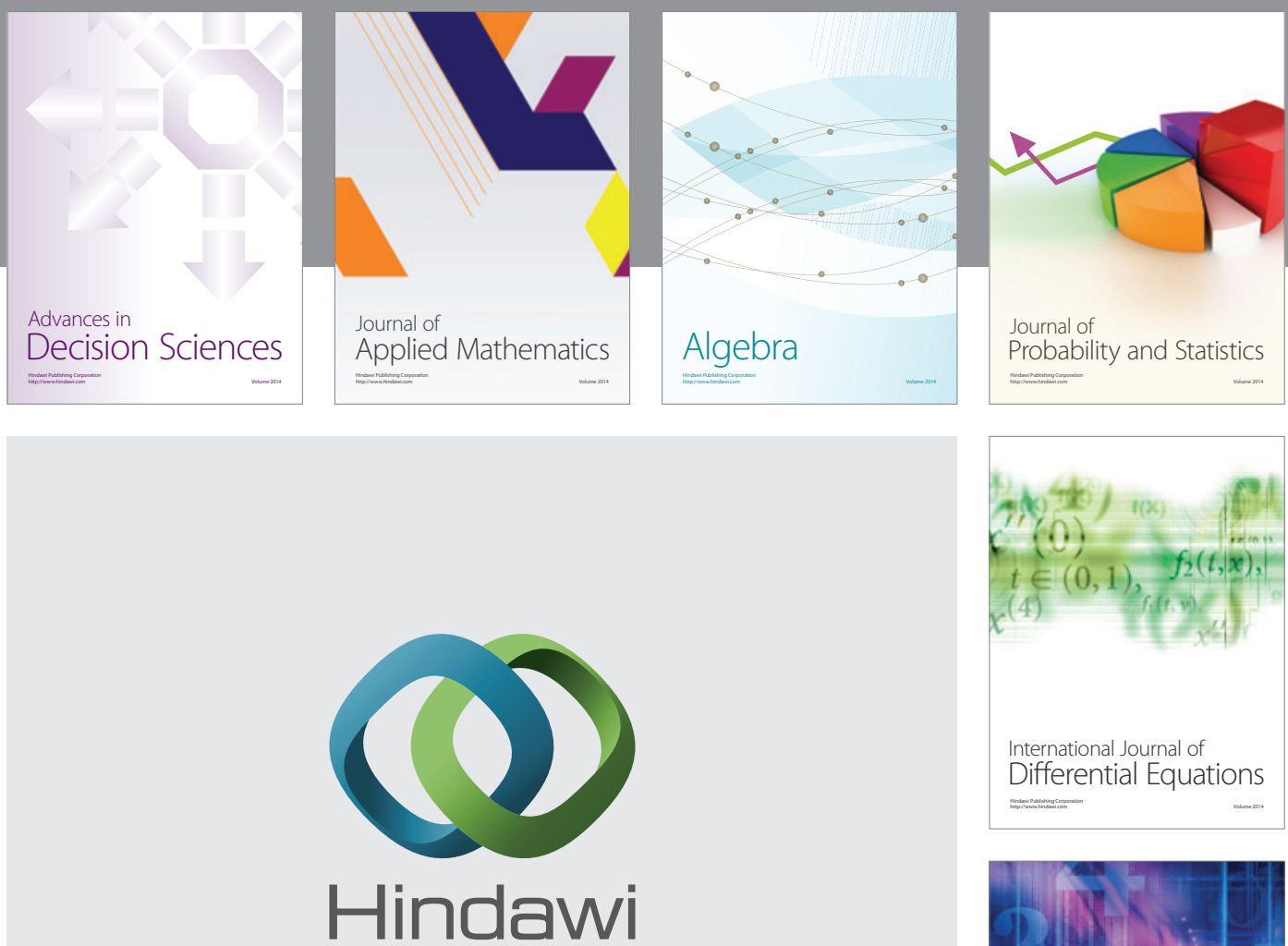

Submit your manuscripts at http://www.hindawi.com
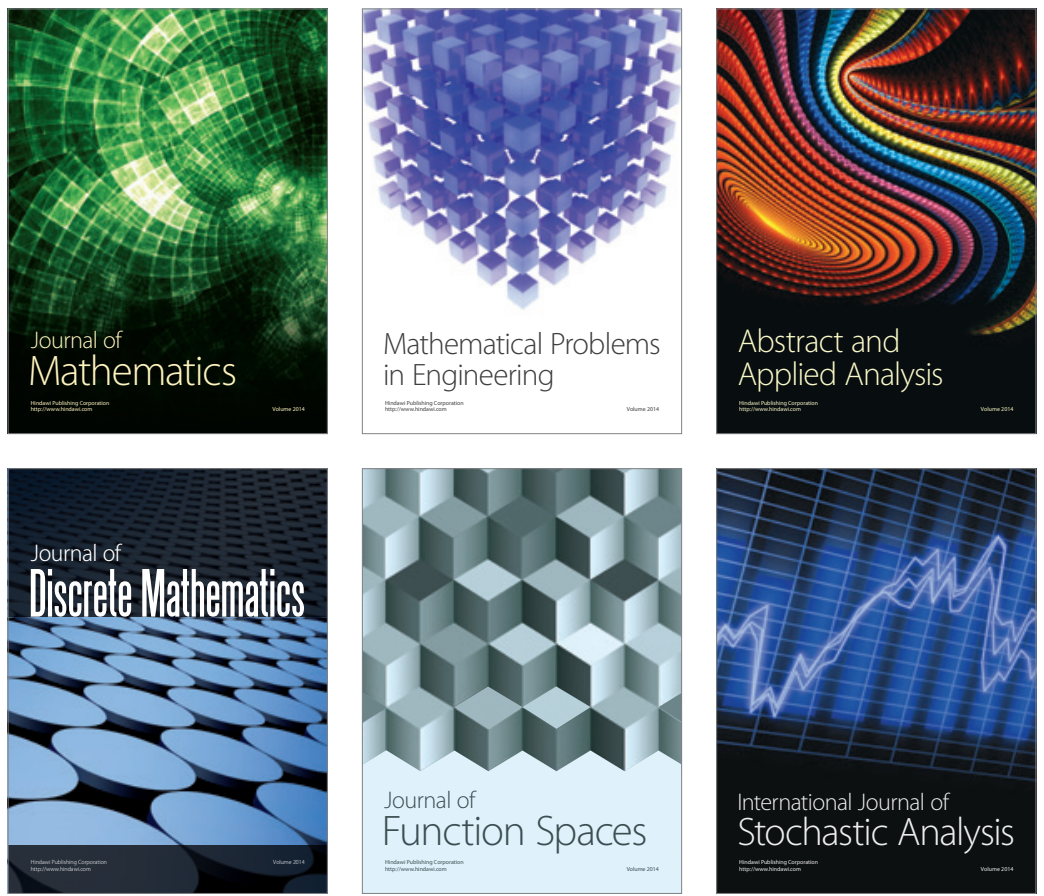

Journal of

Function Spaces

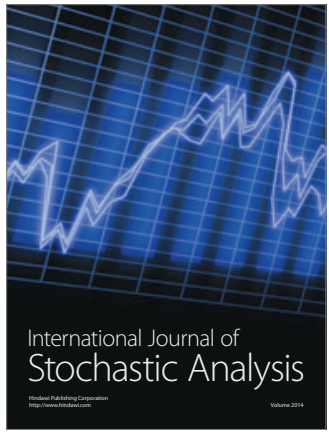

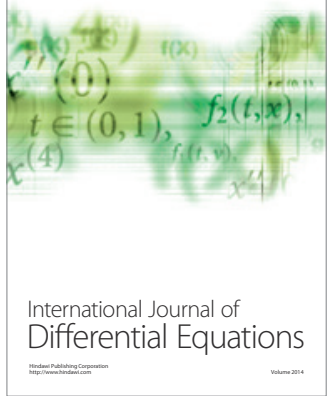
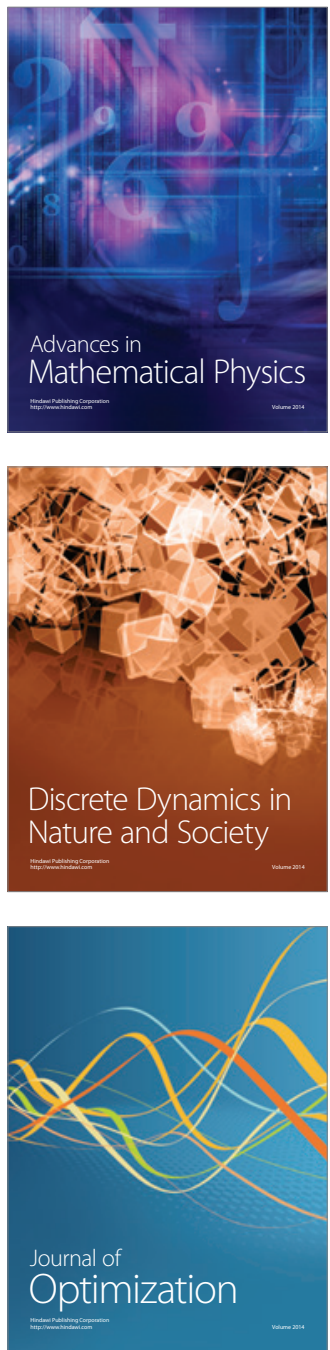